Check for updates

Cite this: RSC Adv., 2017, 7, 47029

Received 19th June 2017

Accepted 2nd October 2017

DOI: $10.1039 / \mathrm{c} 7 \mathrm{ra06810g}$

rsc.li/rsc-advances

\section{Polymer-grafted magnetic microspheres for enhanced removal of methylene blue from aqueous solutions $\uparrow$}

\author{
Bincheng Xu, (D) ab Chaofan Zheng, ${ }^{\text {ab }}$ Huaili Zheng, ${ }^{\text {ab }}$ Yili Wang, ${ }^{c}$ Chun Zhao, ${ }^{\text {ab }}$ \\ Chuanliang Zhao ${ }^{\text {ab }}$ and Shixin Zhang ${ }^{\text {ab }}$
}

Novel polymer-grafted magnetic microspheres (GMMs) were prepared by graft polymerization of 2-acrylamido-2-methylpropane sulfonic acid (AMPS) and acrylic acid (AA) onto the surface of chitosan/ magnetite composite microspheres (MMs). The magnetic microspheres were fully characterized and then applied to the adsorption of a cationic dye (methylene blue, MB) from aqueous solutions. Results show that the adsorption capacity of GMMs was notably enhanced compared with MMs. Furthermore, the effects of initial solution $\mathrm{pH}$, contact time and initial concentration on MB adsorption by GMMs were systematically investigated. The adsorption kinetics and adsorption isotherms are well described by pseudo-second-order kinetic model and Langmuir isotherm model respectively, suggesting the adsorption is a homogeneous monolayer adsorption. The maximum MB adsorption capacity by GMMs is found to be $925.9 \mathrm{mg} \mathrm{g}^{-1}$ at $298.15 \mathrm{~K}$ and initial solution $\mathrm{pH}$ 9.0, as determined from the Langmuir isotherm. The MB-loaded GMMs can be rapidly separated and effectively regenerated at $\mathrm{pH}$ 2.0.

\section{Introduction}

Dyes are widely used in a variety of industries, such as in textiles, foodstuffs, artificial fibers, and in the pharmaceutical and leathers industries, which poses a threat to the environment. ${ }^{1}$ Dyes in waterbodies can reduce the photosynthetic activity, thus affecting symbiotic processes. ${ }^{2}$ Moreover, most dyes have toxic effects on aquatic ecosystems and human life by creating mutagenic, carcinogenic effects and dysfunction of the organs. ${ }^{3}$ However, wastewater containing dyes is usually very difficult to treat, because most dyes are resistant to aerobic digestion and stable to light heat and oxidizing agents due to their stable and complex chemical structures, and low biodegradability. ${ }^{4,5}$ Therefore, it is urgently necessary to develop efficient techniques for the removal of dyes from wastewater before discharge.

Numerous techniques are available for dye removal from wastewater, such as coagulation, ${ }^{6}$ advanced oxidation processes (AOPs), ${ }^{7}$ membrane separation ${ }^{8}$ and adsorption ${ }^{9}$ etc. Among the

${ }^{a}$ Key Laboratory of the Three Gorges Reservoir Region's Eco-Environment, State Ministry of Education, Chongqing University, Chongqing 400045, China. E-mail: zhl@cqu.edu.cn; Fax: +86 23 65120827; Tel: +862365120827

${ }^{b}$ National Centre for International Research of Low-Carbon and Green Buildings, Chongqing University, Chongqing 400045, China

${ }^{c}$ College of Environmental Science and Engineering, Research Center for Water Pollution Source Control and Eco-remediation, Beijing Forestry University, Beijing 100083, China

$\dagger$ Electronic supplementary information (ESI) available. See DOI: $10.1039 / \mathrm{c} 7 \mathrm{ra06810g}$ above techniques, adsorption is considered as a promising technique due to its comparably ease of operation, low cost, insensitivity to toxic substances and effectiveness even in dilute solutions. ${ }^{10}$ As an adsorbent for the removal of dyes from wastewater, activated carbon has been widely used due to its high adsorption capacity. ${ }^{\mathbf{1 1}}$ However, its application is restricted for its high cost and difficulty of regeneration. Therefore, lowcost adsorbents such as biosorbents have been widely developed. ${ }^{12}$

Chitosan (CS) is an appealing bioadsorbent due to its biodegradability, biocompatibility, bioadhesivity, polyfunctionality, hydrophilicity and remarkable adsorption capacity. ${ }^{13}$ However, its application as adsorbent is limited by some shortcomings such as low acid stability and low mechanical strength. ${ }^{\mathbf{1 4}}$ Furthermore, the adsorption performance of raw chitosan without appropriate modification is usually unsatisfactory because functional groups with large adsorption capacity for target dyes are insufficient. Therefore, various modifications such as cross-linking and grafting have been employed to improve the mechanical characteristics and adsorption properties of chitosan. ${ }^{15}$

Moreover, it is inefficient to separate chitosan-based adsorbents from treated water using traditional separation methods such as filtration and sedimentation due to the loss of adsorbents and secondary pollutions. ${ }^{16}$ To overcome these problems, chitosan-based magnetic adsorbents, which can be separated from the aqueous solutions through a simple magnetic process, have been widely developed. ${ }^{17}$ The most available method of chitosan-based magnetic adsorbents preparation is to embed 
$\mathrm{Fe}_{3} \mathrm{O}_{4}$ nanoparticles into cross-linked chitosan microspheres, namely chitosan/magnetite composite microspheres (MMs). ${ }^{18}$ In addition, to introduce more desired functional groups to MMs, low molecular weight compounds are widely grafted onto chitosan backbone. ${ }^{19}$ However, only one layer of functional groups at most are grafted onto the surface of MMs by the above methods. In order for more grafted functional groups, polymer grafting is a better choice. ${ }^{20}$ Dolatkhah A. et al. reported the fabrication of magnetic nanocomposites consisting of polymergrafted chitosan and $\mathrm{Fe}_{3} \mathrm{O}_{4}$ nanoparticles for removal of methylene blue (MB) from aqueous solutions effectively, illustrating that polymer grafting makes a great contribution to the adsorption capacity of magnetic adsorbents. ${ }^{21}$

In this work, polymer-grafted magnetic microspheres (GMMs) were prepared by graft polymerization of 2-acrylamido2-methylpropane sulfonic acid (AMPS) and acrylic acid (AA) onto the surface of MMs and then applied for the adsorption of MB from aqueous solutions. The adsorption performance including the effects of the initial solution $\mathrm{pH}$, adsorption kinetics, isothermal adsorption equilibrium and reusability was investigated. Furthermore, adsorption mechanism is discussed in detail.

\section{Materials and methods}

\subsection{Materials}

Chitosan (CS, $\geq 95.0 \%$ deacetylation, $100-200 \mathrm{mPa}$ s viscosity) was obtained from Cheng Du Micxy Chemical Co. Ltd. Ferrous chloride tetrahydrate $\left(\mathrm{FeCl}_{2} \cdot 4 \mathrm{H}_{2} \mathrm{O}\right)$, ferric chloride hexahydrate $\left(\mathrm{FeCl}_{3} \cdot 6 \mathrm{H}_{2} \mathrm{O}\right)$, hydrochloric acid $(\mathrm{HCl})$ and sodium hydroxide $(\mathrm{NaOH})$ were purchased from Chongqing Chuandong Chemical Co., Ltd. All other chemicals were purchased from Chengdu Kelong Chemical Reagent Co. Ltd. All reagents were used without further purification and Milli-Q ultrapure water $(18.2 \mathrm{M} \Omega \mathrm{cm})$ was used for the preparation of aqueous solutions.

\subsection{Synthesis of magnetic adsorbents}

2.2.1. Synthesis of $\mathrm{MMs}$. $\mathrm{Fe}_{3} \mathrm{O}_{4}$ nanoparticles were synthesized by the chemical co-precipitation method ${ }^{22}$ as described in ESI Text S1. $\uparrow$ The MMs were synthesized according to the reported literature with a slight modification. ${ }^{23}$ Briefly, $7.5 \mathrm{~g}$ chitosan powder was firstly dissolved in $250 \mathrm{~mL} \mathrm{HCl}$ solution $\left(0.5 \%\right.$, w/w). Then, $1.5 \mathrm{~g} \mathrm{Fe}_{3} \mathrm{O}_{4}$ nanoparticles were added to the mixture. The obtained suspension was dispersed evenly in $750 \mathrm{~mL}$ cyclohexane containing $5 \mathrm{~mL}$ Span 80 . Subsequently, $10 \mathrm{~mL}$ glutaraldehyde solution $(50 \%$, w/w) was dropwise added to the water-cyclohexane suspension. The mixture was further stirred for $1 \mathrm{~h}$ at $50{ }^{\circ} \mathrm{C}$. Finally, the product was collected by magnetic separation and repeatedly washed with ethanol and water. The chitosan/magnetite composite microspheres (MMs) were obtained. Moreover, the microspheres without magnetism as a control were also prepared in the same way, coded as Ms.

2.2.2. Synthesis of GMMs. The GMMs were synthesized according to the reported literature with a modification. ${ }^{24}$ The synthesis route was illustrated in Fig. S1. $\dagger$ Briefly, wet MMs
$(50 \mathrm{~g})$ were dispersed in $100 \mathrm{~mL}$ water under $\mathrm{N}_{2}$ atmosphere. Then, $10 \mathrm{~mL}$ of $0.05 \mathrm{~g} \mathrm{~mL}^{-1}$ potassium persulfate (KPS) aqueous solution was added, followed by $\mathrm{N}_{2}$ purging for $5 \mathrm{~min}$. A certain amount of solution containing $1.3 \mathrm{~mL}$ AA and $3.9 \mathrm{~g}$ AMPS monomers was added into the mixture. The reaction was further carried out for $3 \mathrm{~h}$ under $\mathrm{N}_{2}$ atmosphere at $50{ }^{\circ} \mathrm{C}$. Finally, the product was harvested by magnetic separation and washed repeatedly with ethanol and water. The polymer-grafted magnetic microspheres (GMMs) were obtained. Moreover, the polymer-grafted microspheres without magnetism as a control were also prepared in the same way, coded as GMs.

\subsection{Characterization}

The morphology analysis was carried out on a scanning electron microscope (SEM) (S-3400N II, Hitachi, Japan). Size distribution of the microspheres was tested via a particle size analyzer (BT-9300HT, Bettersize, China). Fourier transform infrared (FTIR) spectra were recorded by a FTIR spectrometer (Nicolet iS5, Nicolet, USA) using $\mathrm{KBr}$ pellets. Solid-state ${ }^{13} \mathrm{C}$ nuclear magnetic resonance (NMR) spectra with cross polarization/ magic angle spinning (CP/MAS) of the samples were carried out on a Bruker AVANCE III 400 WB spectrometer equipped with a 9.39 T magnet at $297 \mathrm{~K}$ as described in ESI Text S2. $\dagger$ X-ray photoelectron spectroscopy (XPS) spectra were obtained by using a XPS spectrometer (ESCALAB250Xi, Thermo Fisher Scientific, USA). The thermal decomposition property analysis was conducted by a thermogravimetric analyzer (TGA) (STA-449F3, Netzsch, Germany). X-Ray diffraction (XRD) patterns were obtained by an X-ray diffractometer (DMAX/2C, Japan) with the CuK $\alpha$ radiation $(k=1.54056 \AA)$. Magnetic property analysis was determined by a vibrating sample magnetometer (VSM) (VSM 7410, Lake Shore, USA). Zeta potential measurement was carried out on a Zetasizer Nano ZS90 (Malvern Instruments Ltd., Malvern, UK).

\subsection{Leaching test}

Leaching of Fe from magnetic microspheres at different $\mathrm{pHs}$ was investigated. $0.05 \mathrm{~g}$ magnetic microspheres were dispersed in $50 \mathrm{~mL}$ water at predetermined $\mathrm{pH}$. After $24 \mathrm{~h}$ of shaking, the concentration of $\mathrm{Fe}$ in water was determined by an inductively coupled plasma-optical emission spectroscopy (ICP-OES, Optima 2100 DV, Perkin-Elmer Instruments, USA).

\subsection{Adsorption experiments}

Batch experiments were carried out using a $50 \mathrm{~mL} \mathrm{MB}$ solution with a fixed mass of the adsorbent $(50 \mathrm{mg})$ in a $100 \mathrm{~mL}$ vial. The vials were shaken at $150 \mathrm{rpm}$ at predetermined temperature in a shaking incubator. Sample solutions were taken at predetermined time intervals and filtered with a $0.45 \mu \mathrm{m}$ polytetrafluoroethylene (PTFE) syringe filter (Millipore, USA). The concentration of MB was measured using an UV-visible spectrophotometer (TU-1901, Beijing Purkinje General Instrument Co., Ltd., China) at $665 \mathrm{~nm}$ (wavelength absorption maximum of $\mathrm{MB})$. All experiments were run in triplicate.

The effects of initial solution $\mathrm{pH}$ on the adsorption capacity were investigated from $\mathrm{pH} 2.0$ to 11.0. The initial solution $\mathrm{pH}$ 
was adjusted using $0.1 \mathrm{~mol} \mathrm{~L}{ }^{-1} \mathrm{HCl}$ and/or $0.1 \mathrm{~mol} \mathrm{~L}^{-1} \mathrm{NaOH}$ solution. The initial concentration of $\mathrm{MB}$ solution and contact time were $1000 \mathrm{mg} \mathrm{L}^{-1}$ and $24 \mathrm{~h}$, respectively. The equilibrium adsorption amount of $\mathrm{MB}, q_{\mathrm{e}}\left(\mathrm{mg} \mathrm{g}^{-1}\right)$, was calculated according to the following equation:

$$
q_{\mathrm{e}}=\frac{V\left(C_{0}-C_{\mathrm{e}}\right)}{m}
$$

where $C_{0}$ and $C_{\mathrm{e}}\left(\mathrm{mg} \mathrm{\textrm {L } ^ { - 1 }}\right)$ are the initial and equilibrium concentration of $\mathrm{MB}$ in the solution, respectively. $V(\mathrm{~L})$ is the volume of the dye solution; $m(\mathrm{~g})$ is the weight of the adsorbent.

The adsorption kinetics experiments were performed using $1000 \mathrm{mg} \mathrm{L}^{-1}$ as initial concentration of $\mathrm{MB}$ and the initial solution $\mathrm{pH}$ was adjusted to 9.0. $1 \mathrm{~mL}$ of sample solutions were collected at desired time intervals to analyze MB concentration. The adsorption amount of dye at time $t_{i}, q\left(t_{i}\right)\left(\mathrm{mg} \mathrm{g}^{-1}\right)$ was calculated using the following equation:

$$
q\left(t_{i}\right)=\frac{\sum_{i=1}^{n}\left(C_{t_{i-1}}-C_{t_{i}}\right) V_{t_{i-1}}}{m}
$$

where $C_{t_{0}}\left(C_{0}\right)$ and $C_{t_{i}}\left(\mathrm{mg} \mathrm{L}^{-1}\right)$ are the initial MB concentration and $\mathrm{MB}$ concentration at time $t_{i}$, respectively. $V_{t_{i}}(\mathrm{~L})$ is the volume of the residual solution at time $t_{i}$, and $m(\mathrm{~g})$ is the weight of the adsorbent.

The isothermal adsorption equilibrium experiments were conducted with solutions of different initial concentrations of $\mathrm{MB}$ in a range of $100-1200 \mathrm{mg} \mathrm{L}^{-1}$. The initial solution $\mathrm{pH}$ was adjusted to 9.0 and the contact time was $24 \mathrm{~h}$. The equilibrium adsorption amount of MB was calculated based on eqn (1).

\subsection{Desorption and regeneration experiments}

First, $50 \mathrm{mg}$ GMMs were immersed into a $100 \mathrm{~mL}$ vial containing $50 \mathrm{~mL} \mathrm{MB}$ solution with the initial concentration of $1000 \mathrm{mg} \mathrm{L}^{-1}$ and initial solution $\mathrm{pH}$ of 9.0. The vial was shaken for $24 \mathrm{~h}$ at $150 \mathrm{rpm}$ at $25^{\circ} \mathrm{C}$ in a shaking incubator for $\mathrm{MB}$ adsorption after which sample solutions were taken and measured by the same method described in Section 2.5. The MB-loaded microspheres were magnetically separated and then added into a $100 \mathrm{~mL}$ vial containing $50 \mathrm{~mL}$ of $0.01 \mathrm{~mol} \mathrm{~L}^{-1} \mathrm{HCl}$ solution. The vial was shaken for $24 \mathrm{~h}$ at $150 \mathrm{rpm}$ at $25^{\circ} \mathrm{C}$ in a shaking incubator for MB desorption. Finally, the desorbed microspheres were collected by a magnet, washed with water repeatedly, and then subsequently reused in the next cycle of adsorption. The adsorption-desorption experiments were conducted for five cycles.

\section{Results and discussion}

\subsection{Synthesis of the adsorbents}

The resulting GMMs were prepared by a two-step method. In the first step, MMs were prepared by a water/oil (W/O) emulsion crosslinking technique. The $\mathrm{W} / \mathrm{O}$ emulsion was prepared by adding the suspension containing chitosan and $\mathrm{Fe}_{3} \mathrm{O}_{4}$ nanoparticles into a dispersion medium composed of cyclohexane. Glutaraldehyde was used as a crosslinking agent. In the second step, poly(AMPS-AA) chains were grafted onto cross-linked chitosan backbone using a grafting from approach. KPS was used as an initiator to generate free sulfate radical $\left(\mathrm{SO}_{4}{ }^{-\bullet}\right)$, resulting in polymerization via the amino groups. The chemical reactions of the preparation process are described in Scheme 1.

\subsection{Characteristics of the adsorbents}

3.2.1. FTIR spectra. Fig. 1 depicts the FTIR spectra of CS, MMs, and GMMs. For CS, the absorption peaks at 3447, 1603, 1155 and $1079 \mathrm{~cm}^{-1}$ are attributed to the hydroxyl $(\mathrm{O}-\mathrm{H})$, amino group $(\mathrm{N}-\mathrm{H})$, glucosidic bond $(\mathrm{C}-\mathrm{O}-\mathrm{C})$ and the primary alcohol $(\mathrm{C}-\mathrm{OH})$ in $\mathrm{CS}$, respectively. ${ }^{25}$ For both spectra of MMs and GMMs, the peaks at $1636 \mathrm{~cm}^{-1}$ correspond to $\mathrm{N}-\mathrm{H}$ stretching vibration and imine linkage $(\mathrm{C}=\mathrm{N})$ that formed between amine groups of chitosan and aldehyde groups of glutaraldehyde. ${ }^{26}$ The peaks at $1715,1550 \mathrm{~cm}^{-1}$ in the spectrum of GMMs are assigned to residual free carboxylic groups $(-\mathrm{COOH})$ and carboxylate ions $\left(-\mathrm{COO}^{-}\right)$respectively, indicating the successful grafting of AA. ${ }^{23}$ Furthermore, the peaks at 1041 and $625 \mathrm{~cm}^{-1}$ in the spectrum of GMMs are attributed to the stretching vibration of $\mathrm{S}=\mathrm{O}$ and $\mathrm{S}-\mathrm{O}$ respectively, confirming the successful grafting of AMPS. ${ }^{27}$

3.2.2. Solid-state ${ }^{13} \mathrm{C}$ NMR spectra. The solid-state ${ }^{13} \mathrm{C}$ NMR spectra of Ms and GMs are presented in Fig. S2 $\uparrow$ and the signals associated with the monomeric form of chitosan is demonstrated..$^{28}$ For Ms, the peaks at 27.5 and $40.3 \mathrm{ppm}$ are attributed to the methylene groups of glutaraldehyde with different chemical environments. The peaks at 125-144 ppm are assigned to conjugated ethylenic bond and the one at $176 \mathrm{ppm}$ to imine bond. ${ }^{29}$ Furthermore, the peak at $93.8 \mathrm{ppm}$ is related to the ${ }^{13} \mathrm{C}$ shift variation of $\mathrm{C} 1$ peak. By comparison, the spectral differences between GMs and Ms associate with the broadening and ${ }^{13} \mathrm{C}$ shift variation, along with new peaks at $27.5 \mathrm{ppm}$, $36.3 \mathrm{ppm}, 41.6 \mathrm{ppm}, 52.6 \mathrm{ppm}, 60.9 \mathrm{ppm}$ and $175.8 \mathrm{ppm}$. The assignments of these peaks are given in Table S1. $\dagger^{\mathbf{3 0 , 3 1}}$ All these spectra indicate the successful grafting of both AMPS and AA.

3.2.3. XPS spectra. The XPS fully scanned spectra of the magnetic microspheres are shown in Fig. 2. It can be seen that $\mathrm{Al}$ and $\mathrm{Si}$ exist in all the three samples due to adventitious contamination. For $\mathrm{MMs}, \mathrm{Cl}$ is a residual element from the precursor solution. Compared with MMs, the existence of $\mathrm{S}$ element in GMMs confirms the successful grafting of AMPS. It is worth mentioning that no Fe exists in both magnetic microspheres due to the chitosan coating on the surface of $\mathrm{Fe}_{3} \mathrm{O}_{4}$ nanoparticles. Furthermore, the XPS spectra of $\mathrm{C} 1 \mathrm{~s}$ for the magnetic microspheres are presented in Fig. 3. Three peaks appear on the $\mathrm{C}$ 1s spectra of MMs, the peak at $284.2 \mathrm{eV}$ corresponding to $\mathrm{C}-\mathrm{C}$ or adventitious carbon, the peak at $286.0 \mathrm{eV}$ corresponding to $\mathrm{C}-\mathrm{N}, \mathrm{C}=\mathrm{N}, \mathrm{C}-\mathrm{O}$ or $\mathrm{C}-\mathrm{O}-\mathrm{C}$, the peak at $288.1 \mathrm{eV}$ corresponding to $\mathrm{C}=\mathrm{O}$ or $\mathrm{O}-\mathrm{C}-\mathrm{O} .{ }^{32}$ For $\mathrm{GMMs}$, a new peak appears at $288.6 \mathrm{eV}$ attributing to $\mathrm{O}-\mathrm{C}=\mathrm{O}$, indicating the successful grafting of AA. ${ }^{33}$

3.2.4. XRD patterns. The XRD patterns of $\mathrm{Fe}_{3} \mathrm{O}_{4}, \mathrm{MMs}$, GMMs, Ms and GMs are shown in Fig. S3. $\dagger$ For $\mathrm{Fe}_{3} \mathrm{O}_{4}$, several diffraction peaks corresponding to (111), (220), (311), (400), (422), (511) and (440) planes of the iron oxide respectively, are 


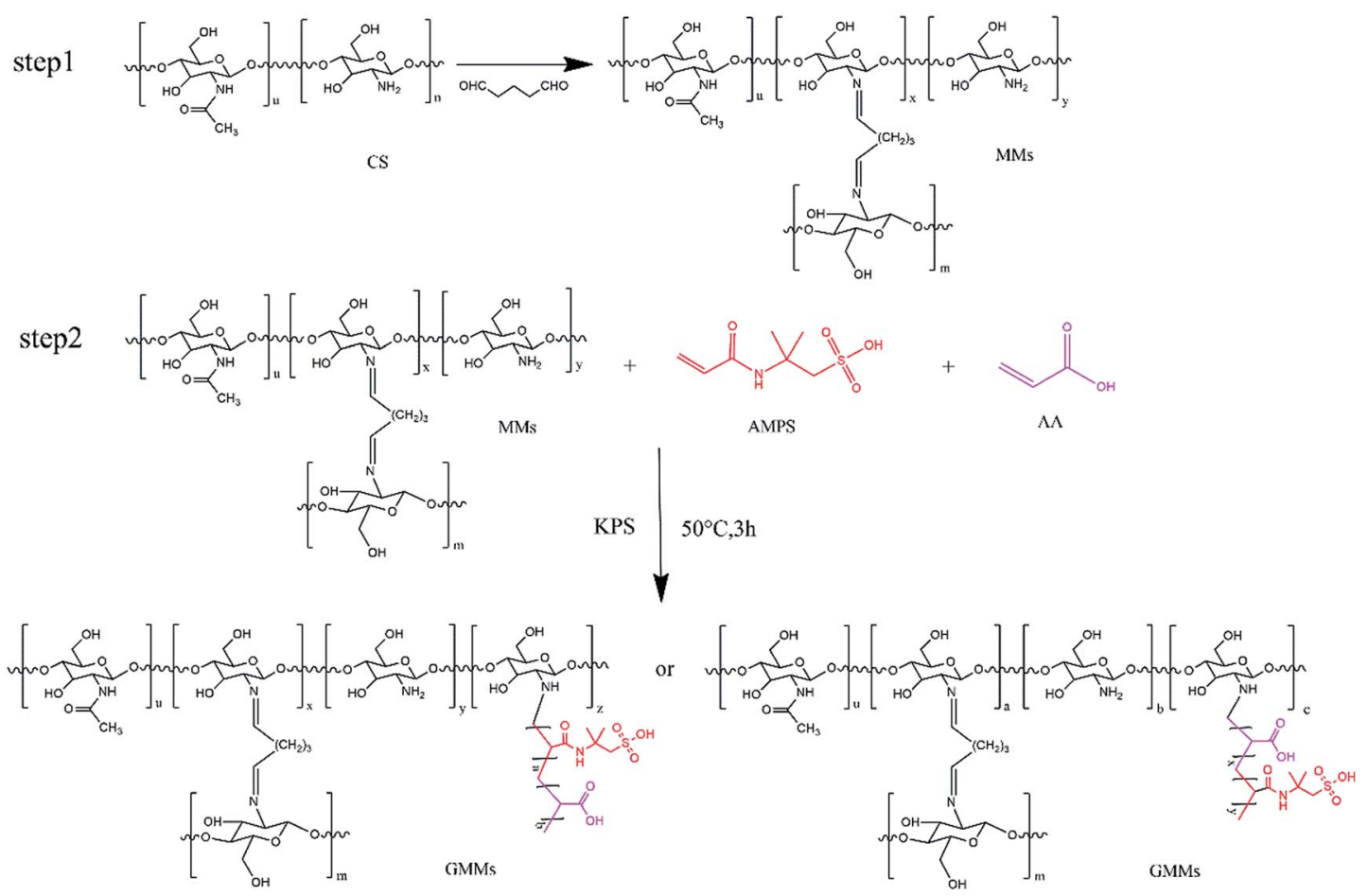

Scheme 1 Chemical reactions of the preparation process of MMs and GMMs.

observed. ${ }^{34}$ The characteristic peaks of $\mathrm{Fe}_{3} \mathrm{O}_{4}$ are observed in the patterns of both magnetic microspheres, indicating the crystal phases of $\mathrm{Fe}_{3} \mathrm{O}_{4}$ were maintained after the formation of the magnetic microspheres. ${ }^{21}$ In addition, broad peaks $\left(\sim 25^{\circ}\right.$ for Ms and MMs; $\sim 21^{\circ}$ for GMs and GMMs) are observed, indicating the amorphous structure of organic fraction. ${ }^{35}$

3.2.5. SEM images and size distribution. The microstructure and surface morphology of the magnetic microspheres are shown in Fig. 4. It is found that MMs and GMMs are both spherical microspheres. The size distribution of the magnetic microspheres is shown in Fig. S4. $\dagger$ The diameter of GMMs is larger than that of MMs due to the polymer grafting. The presence of abundant sulfonic $\left(-\mathrm{SO}_{3} \mathrm{H}\right)$ and carboxyl $(-\mathrm{COOH})$ groups on the grafted polymer is expected to enhance the adsorption capacity of GMMs toward dyes. ${ }^{36}$

3.2.6. TG analysis. The thermal stability of the magnetic microspheres is shown in Fig. S5. $\dagger$ Since the organic matters in both microspheres would be fully thermo-decomposed before $750{ }^{\circ} \mathrm{C},{ }^{37}$ the curves indicate that the total content of polymeric matters in magnetic microspheres for GMMs is $90.68 \%$, which is higher than that for MMs approximate $86.53 \%$. It is due to the fact that the polymer has been grafted onto MMs.

3.2.7. Magnetic characterization. Fig. 5 shows the magnetization curves of MMs and GMMs. The invisible hysteresis loop

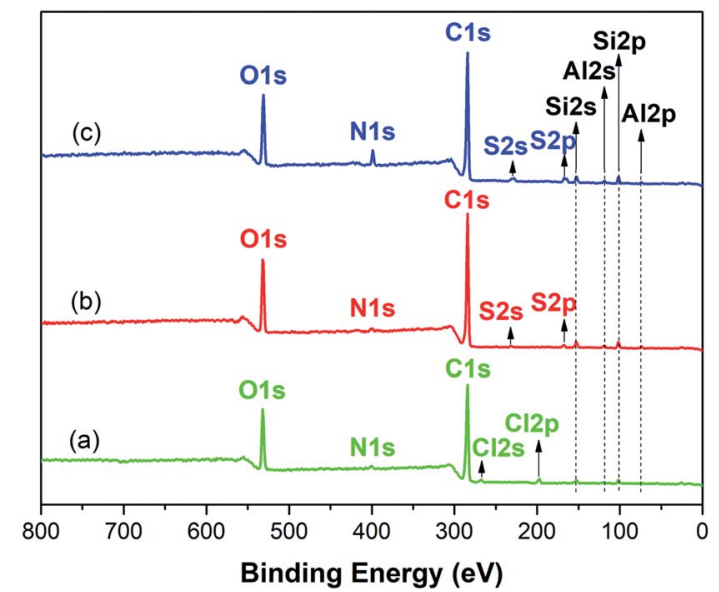

Fig. 2 XPS fully scanned spectra of MMs (a), GMMs (b) and MB-loaded GMMs (c).

Fig. 1 FTIR spectra of CS (a), MMs (b) and GMMs (c). 

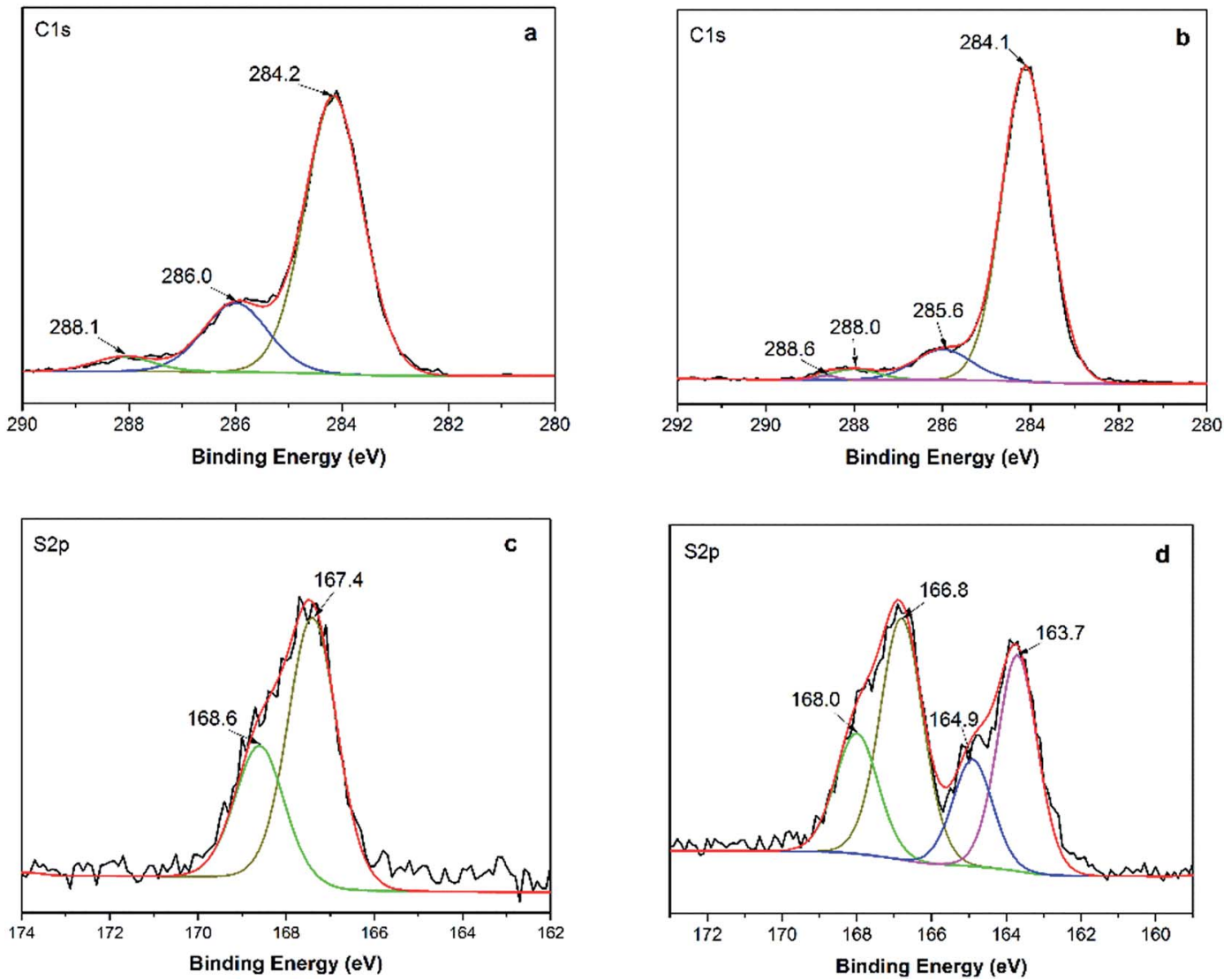

Fig. 3 XPS spectra of C 1s for MMs (a) and GMMs (b); XPS spectra of S 2p for GMMs (c) and MB-loaded GMMs (d).

of each curve demonstrates typical paramagnetic properties of both microspheres. ${ }^{38}$ The saturation magnetization value was measured to be $55.9 \mathrm{emu} \mathrm{g}^{-1}$ for $\mathrm{Fe}_{3} \mathrm{O}_{4}$ (not shown in Fig. 5), $10.82 \mathrm{emu} \mathrm{g}^{-1}$ for MMs and $5.05 \mathrm{emu} \mathrm{g}^{-1}$ for GMMs. Although the saturation magnetization decreased after chitosan coating and polymer grafting, the relatively high saturation magnetization of GMMs significantly facilitates separation and regeneration of the magnetic adsorbent. Furthermore, the inset image in Fig. 5 illustrates the magnetic separation of MB-loaded microspheres from aqueous solution under the application of
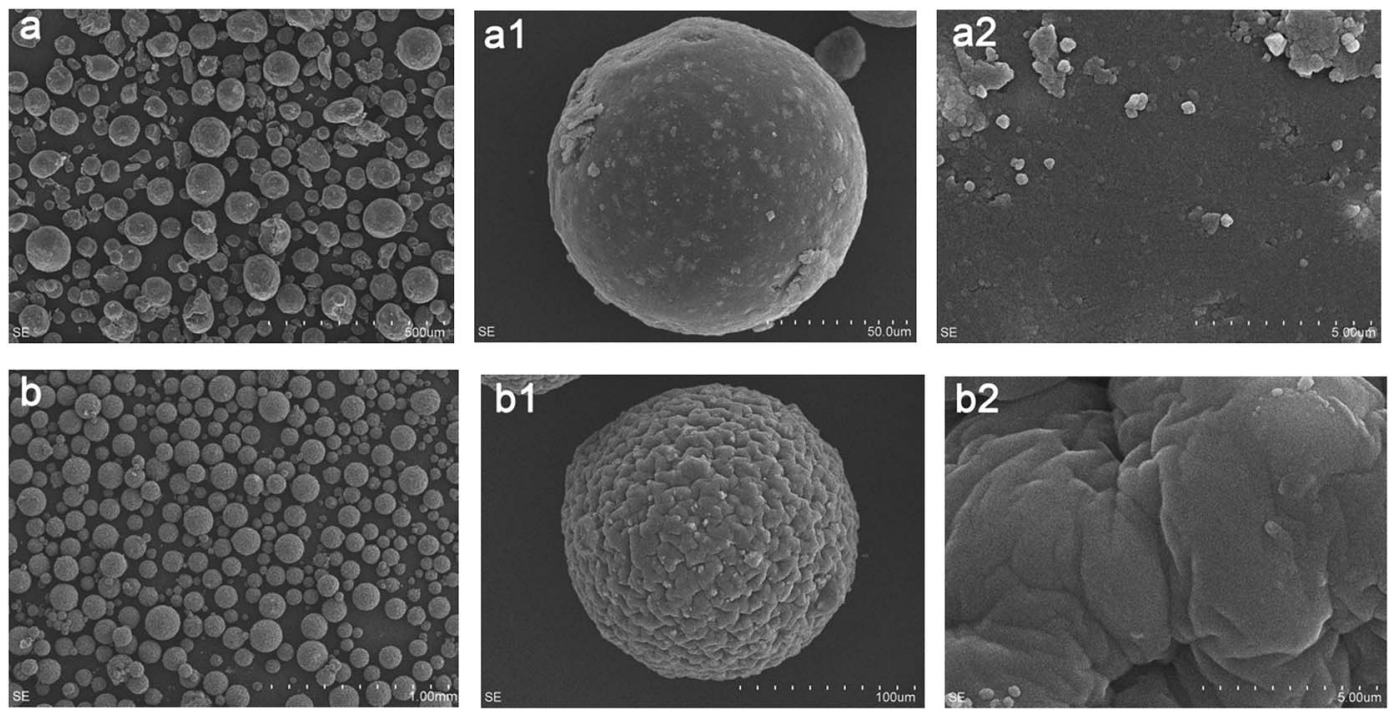

Fig. 4 SEM images of MMs (a, a1, a2) and GMMs (b, b1, b2). 


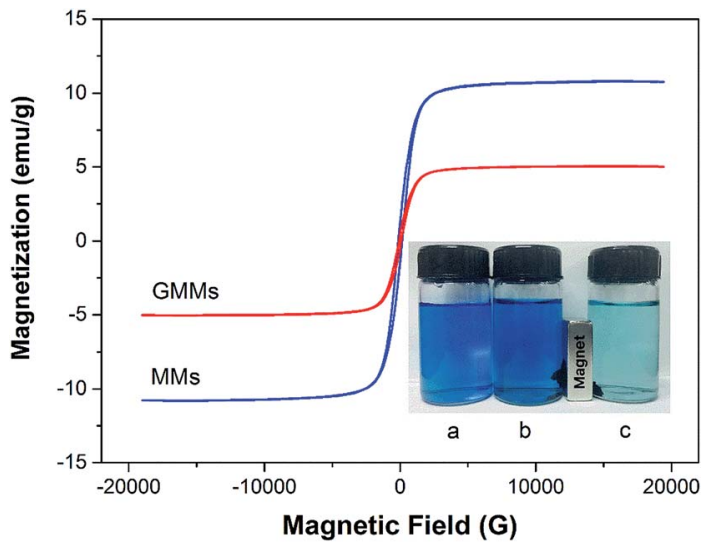

Fig. 5 Magnetization curves of MMs and GMMs. The insert is the photographs of MB solutions before (a) and after adsorption using MMs (b) and GMMs (c) including the effects of actual magnetic separation. The initial concentration of $M B$ is $20 \mathrm{mg} \mathrm{L}^{-1}$ and the initial solution $\mathrm{pH}$ is 9.0 .

an external magnetic field, indicating sufficient magnetic strength of the magnetic microspheres.

\subsection{Effects of initial solution $\mathbf{p H}$}

The $\mathrm{pH}$ value of the solution is an important factor in the adsorption process as it influences not only the conversion of the adsorbates but also the surface properties of the adsorbent and ionization/dissociation of the functional groups. ${ }^{39}$ The effects of initial solution $\mathrm{pH}$ on the adsorption of MB onto MMs and GMMs are illustrated in Fig. S6. $\dagger$ The adsorption capacity of GMMs is notably greater than that of MMs and increases with increasing solution $\mathrm{pH}$. As displayed in Fig. 6, zeta potential of GMMs is less than zero due to the deprotonation of sulfonic $\left(-\mathrm{SO}_{3} \mathrm{H}\right)$ groups at $\mathrm{pH}$ of 2.0 (above the $\mathrm{p} K_{\mathrm{a}}$ value of AMPS $\left.=1.5\right)$. Therefore, the noticeable adsorption capacity at $\mathrm{pH} 2.0$ can be attributed to electrostatic interactions between the deprotonated sulfonic $\left(-\mathrm{SO}_{3} \mathrm{H}\right)$ groups and positively charged $\mathrm{MB}$ molecules. When $\mathrm{pH}$ is larger than the $\mathrm{p} K_{\mathrm{a}}$ of $\mathrm{AA}$ which is 4.2, both the carboxyl $(-\mathrm{COOH})$ and sulfonic $\left(-\mathrm{SO}_{3} \mathrm{H}\right)$ groups of the grafted polymer are ionized, which significantly enhance the

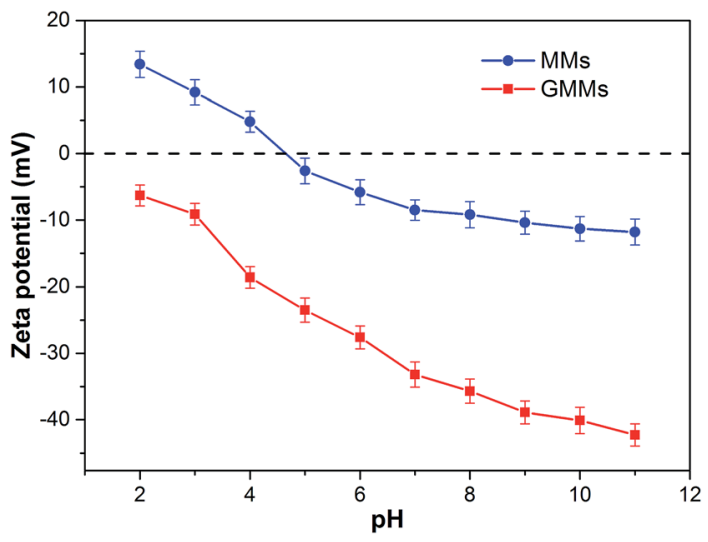

Fig. 6 Zeta potentials-pH profiles of MMs and GMMs. electrostatic interactions between $\mathrm{MB}$ cation and negatively charged grafted polymer. Therefore, it is suggested that electrostatic interaction is one of adsorption mechanisms for the removal of MB by GMMs.

Interestingly, there is a sharp increase of adsorption capacity at the initial solution $\mathrm{pH}$ range from 7.0 to 10.0 where grafted polymer is completely deprotonated. Similar adsorption performance of chitosan-AMPS hydrogel toward MB was reported in previous literature..$^{40}$ As seen in Fig. S7, $\uparrow$ MB has a heteroatomic structure and it tends to form face-to-face dimers through stacking interactions to minimize their hydrophobic interactions with water. ${ }^{41} \mathrm{At}$ high $\mathrm{pH}, \mathrm{MB}$ cations start to loose positive charges, which increases their aggregation. Furthermore, the self-organization of $\mathrm{MB}$ aggregates is promoted by forces such as dispersion forces due to the interaction between $\pi$-systems of the MB and hydrophobic forces of the polyanion. ${ }^{42}$ The grafted polymer containing AMPS exhibit hydrophobic character due to the aliphatic branches on its pendant groups. Consequently, the hydrophobic interactions between the hydrophobic moieties of AMPS and MB aggregates are involved in MB adsorption by GMMs.

From the above, both electrostatic and hydrophobic interactions contribute to the adsorption of MB onto GMMs. Furthermore, the successful adsorption is confirmed by XPS spectra in Fig. 3. In the $\mathrm{S} 2 \mathrm{p}$ spectrum for GMMs (Fig. 3c), sub-bands at 167.4 and $168.6 \mathrm{eV}$ correspond to $2 \mathrm{p}_{3 / 2}$ and $2 \mathrm{p}_{1 / 2}$ peaks of $-\mathrm{SO}_{3}{ }^{-}$groups, respectively. For the S 2p spectrum for MB-loaded GMMs (Fig. 3d), new sub-bands at 163.7 and $164.9 \mathrm{eV}$ are assigned to $2 \mathrm{p}_{3 / 2}$ and $2 \mathrm{p}_{1 /}$ ${ }_{2}$ peaks of the MB adsorbed on GMMs, respectively. ${ }^{43}$

\subsection{Adsorption kinetics}

The results of the adsorption kinetics of MB adsorption onto GMMs are shown in Fig. 7a. The adsorption capacity of MB increases rapidly over the first $50 \mathrm{~min}$ and slows down thereafter. The initial rapid adsorption is attributed to the accessibility of the negatively charged adsorption sites on the grafted polymer. The slower adsorption is attributed to the occupancy of the adsorption sites and reduced osmotic pressure. ${ }^{21}$

Three kinetic models, pseudo-first order (PFO), pseudosecond order (PSO), and intraparticle diffusion models are used to investigate the kinetics involved in the adsorption of $\mathrm{MB}$ by GMMs.

The PFO model assumes that the sorption rate linearly declines with the increase of adsorption capacity. The PSO model, which is usually used to describe a chemical adsorption, assumes that the rate-limiting step is the chemical interaction between the adsorbent and adsorbate.

The PFO and PSO models are expressed in nonlinear forms as eqn (3) and (4) ${ }^{44}$ respectively:

$$
\begin{gathered}
q_{t}=q_{\mathrm{e}}\left(1-\mathrm{e}^{-k_{1} t}\right) \\
q_{t}=\frac{q_{\mathrm{e}}^{2} k_{2} t}{q_{\mathrm{e}} k_{2} t+1}
\end{gathered}
$$

where $q_{\mathrm{e}}\left(\mathrm{mg} \mathrm{g}^{-1}\right)$ and $q_{t}\left(\mathrm{mg} \mathrm{g}^{-1}\right)$ are the adsorption capacity of the adsorbents at the equilibrium and at time $t$ (min), 

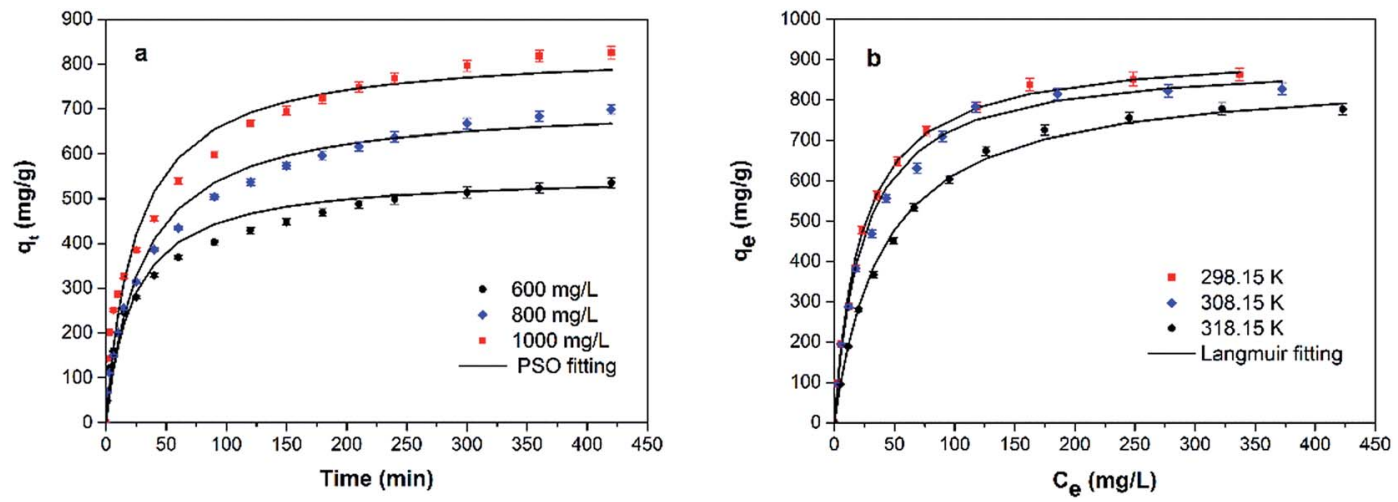

Fig. 7 Adsorption kinetics of MB adsorption onto GMMs at different initial concentrations (a) and adsorption isotherms of MB adsorption onto GMMs at different temperatures (b). The initial solution $\mathrm{pH}$ is 9.0 .

respectively. $k_{1}\left(\mathrm{~min}^{-1}\right)$ and $k_{2}\left(\mathrm{~g} \mathrm{mg}^{-1} \mathrm{~min}^{-1}\right)$ are the rate constant of first-order and second-order adsorption, respectively.

The intraparticle diffusion, which suggests that intraparticle diffusion is the rate-limiting step in the adsorption, is described as follows: $:^{45}$

$$
q_{t}=k_{\mathrm{p}} t^{0.5}+C
$$

where $k_{\mathrm{p}}\left(\mathrm{mg} \mathrm{g}^{-1} \mathrm{~min}^{-0.5}\right)$ is the intraparticle diffusion rate constant, and $C\left(\mathrm{mg} \mathrm{g}^{-1}\right)$ is another constant.

All the experimental data were fitted by aforementioned kinetics models, and the resultant parameters were all listed in Table 1. The correlation coefficients of PSO model are much closer to 1.0 compared to those of other two models and $q$ values calculated from PSO model are closer to those from the experiments. It confirms that chemical adsorption is the rate-controlling step and the adsorption rate depends on the concentration of MB at the surface of the adsorbent. ${ }^{46}$

\subsection{Adsorption isotherms}

Fig. $7 \mathrm{~b}$ depicts the isotherm results for the adsorption of $\mathrm{MB}$ onto GMMs at different temperatures. The obtained experimental data are analyzed based on Langmuir, Freundlich and Dubinin-Radushkevich (D-R) isotherm models, respectively.

The Langmuir isotherm model is based on the assumption that the surface of the adsorbent is homogeneous and all adsorption sites are equivalent, resulting in a monolayer adsorption on a homogeneous surface. In contrast, the Freundlich isotherm model is based on the assumption that different types of adsorption sites are available on the surface, resulting in multilayer heterogeneous adsorptions. ${ }^{47}$

The Langmuir and Freundlich isotherm models can be expressed as eqn (6) and $(7),{ }^{48}$ respectively:

$$
\begin{gathered}
q_{\mathrm{e}}=\frac{q_{\mathrm{m}} K_{\mathrm{L}} C_{\mathrm{e}}}{1+K_{\mathrm{L}} C_{\mathrm{e}}} \\
q_{\mathrm{e}}=K_{\mathrm{F}} C_{\mathrm{e}}{ }^{1 / n}
\end{gathered}
$$

where $q_{\mathrm{e}}\left(\mathrm{mg} \mathrm{g}^{-1}\right)$ and $C_{\mathrm{e}}\left(\mathrm{mg} \mathrm{L}^{-1}\right)$ are the adsorption capacity and concentration of $\mathrm{MB}$ at equilibrium respectively; $q_{\mathrm{m}}\left(\mathrm{mg} \mathrm{g}^{-1}\right)$ is the Langmuir constant related to the maximum adsorption capacity $\left(\mathrm{mg} \mathrm{g}^{-1}\right)$ of the adsorbent; and $K_{\mathrm{L}}\left(\mathrm{L} \mathrm{mg}^{-1}\right)$ is the Langmuir isotherm constant. $K_{\mathrm{F}}$ is the Freundlich isotherm constant, and $n$ (dimensionless) is the heterogeneity factor.

The $\mathrm{D}-\mathrm{R}$ isothermal model is employed to determine the nature of the biosorption processes and can be described as follows: ${ }^{49}$

$$
\ln q_{\mathrm{e}}=\ln Q_{\mathrm{D}}-K_{\mathrm{D}} \varepsilon^{2}
$$

where $Q_{\mathrm{D}}\left(\mathrm{mg} \mathrm{g}^{-1}\right)$ is the theoretical saturation capacity in the $\mathrm{D}-\mathrm{R}$ model, $K_{\mathrm{D}}$ is the constant related to the mean free energy of adsorption, and $\varepsilon$ is the Polanyi potential $\left(\varepsilon=R T \ln \left(1+\frac{1}{C_{\mathrm{e}}}\right)\right)$.

Table 1 Kinetic parameters for MB adsorption onto GMMs at $298.15 \mathrm{~K}$ and initial solution $\mathrm{pH} 9.0$

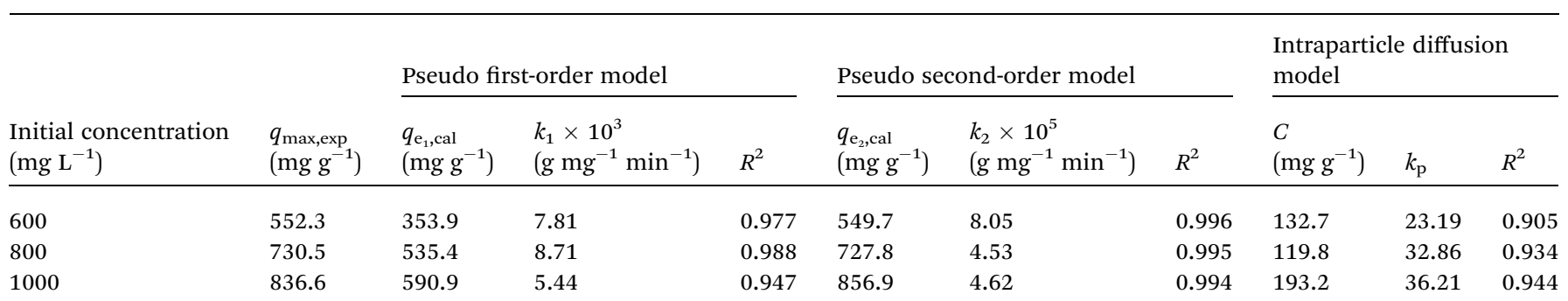


Table 2 Isotherm parameters for MB adsorption onto GMMs at initial solution pH 9.0

\begin{tabular}{|c|c|c|c|c|c|c|c|c|c|c|}
\hline$T(\mathrm{~K})$ & \multicolumn{4}{|c|}{ Langmuir model } & \multicolumn{3}{|c|}{ Freundlich model } & \multicolumn{3}{|l|}{ D-R model } \\
\hline 308.15 & 0.043 & 888.9 & 0.023 & 0.997 & 96.11 & 2.41 & 0.919 & 597.3 & 2.89 & 0.698 \\
\hline 318.15 & 0.025 & 866.6 & 0.039 & 0.998 & 64.37 & 2.18 & 0.926 & 581.8 & 9.42 & 0.678 \\
\hline
\end{tabular}

The final simulated parameters are all listed in Table 2. The correlation coefficients of the Langmuir model are much closer to 1.0 and higher than those of other two models, suggesting that a monolayer adsorption of $\mathrm{MB}$ occurs onto the grafted polymer of GMMs.

Furthermore, the separation factor $\left(R_{\mathrm{L}}\right)$ related to Langmuir model was applied to evaluate the feasibility of adsorption process. It can be calculated by the following equation: ${ }^{50}$

$$
R_{\mathrm{L}}=\frac{1}{1+K_{\mathrm{L}} C_{0}}
$$

where $C_{0}\left(\mathrm{mg} \mathrm{L}^{-1}\right)$ is initial dye concentration and $K_{\mathrm{L}}\left(\mathrm{L} \mathrm{mg}^{-1}\right)$ is Langmuir isotherm constant. The value of $R_{\mathrm{L}}$ indicates the tendency of the adsorption process: irreversible $\left(R_{\mathrm{L}}=0\right)$, favorable $\left(0<R_{\mathrm{L}}<1\right)$, linear $\left(R_{\mathrm{L}}=1\right)$, unfavorable $\left(R_{\mathrm{L}}>1\right)$.

The $R_{\mathrm{L}}$ values of $\mathrm{MB}$ adsorption onto GMMs are much smaller than 1.0, indicating the favorable adsorption for MB.

Moreover, from the Langmuir isotherm model, the maximum adsorption capacity of GMMs for MB at 298.15 K and initial solution pH 9.0 is found to be $925.9 \mathrm{mg} \mathrm{g}^{-1}$ (shown in Table 2), which is notably higher than many other reported values for other adsorbents (shown in Table S2†).

\subsection{Desorption and reusability}

Reuse of adsorbents is of great importance in practical applications. Based on $\mathrm{pH}$ effects on the $\mathrm{MB}$ adsorption as discussed in section 3.3, MB-loaded GMMs would be regenerated under acidic conditions. However, $\mathrm{Fe}_{3} \mathrm{O}_{4}$ can dissolve out from GMMs at $\mathrm{pH}<2.0$ as illustrated in Fig. S8. $\dagger$ Therefore, MB-loaded GMMs were regenerated at $\mathrm{pH} 2.0$ and the regenerated microspheres were reused in the next adsorption cycle. As shown in Fig. S9, $\uparrow$ high adsorption capacity maintains over five cycles and $86.1 \%$ of the original adsorption capacity is preserved after the fifth cycle, indicating the ease of regeneration and moderate reusability of GMMs. The adsorption capacity decreases due to the fact that MB adsorbed at the bottom (near the MMs) of the grafted polymer chains are difficult to desorbed.

\section{Conclusions}

2-Acrylamido-2-methylpropane sulfonic acid (AMPS) and acrylic acid (AA) were successfully grafted onto the surface of chitosan/ magnetite composite microspheres (MMs) by surface-initiated radical polymerization. The polymer-grafted magnetic microspheres (GMMs) show notably enhanced MB adsorption capacity compared with MMs. The excellent MB adsorption is attributed to the hydrophobic and electrostatic interactions between MB cation and negatively charged polymer chains. Adsorption isotherms results suggest that a monolayer adsorption of MB occurs onto the grafted polymer of GMMs. Besides, the MB-loaded GMMs were separated from aqueous solutions quickly under an applied external field and efficiently regenerated and reused over five recycles, indicating that GMMs could be a promising adsorbent for $\mathrm{MB}$ removal in practical applications. Future developments would require a more detailed cost analysis of the materials and the chemical stability as part of the recycle process for these GMMs materials.

\section{Conflicts of interest}

There are no conflicts of interest to declare.

\section{Acknowledgements}

The authors gratefully acknowledge the financial support of the National Natural Science Foundation of China (Project No. 21677020).

\section{References}

1 W. Song, B. Gao, X. Xu, L. Xing, S. Han, P. Duan, W. Song and R. Jia, Bioresour. Technol., 2016, 210, 123-130.

2 A. S. Patra, S. Ghorai, S. Ghosh, B. Mandal and S. Pal, J. Hazard. Mater., 2016, 301, 127-136.

3 Markandeya, N. Dhiman, S. P. Shukla and G. C. Kisku, J. Cleaner Prod., 2017, 149, 597-606.

4 S. P. D. Monte Blanco, F. B. Scheufele, A. N. Módenes, F. R. Espinoza-Quiñones, P. Marin, A. D. Kroumov and C. E. Borba, Chem. Eng. J., 2017, 307, 466-475.

5 M.-T. Nguyen-Le and B.-K. Lee, Chem. Eng. J., 2015, 281, 2033.

6 A. K. Verma, R. R. Dash and P. Bhunia, J. Environ. Manage, 2012, 93, 154-168.

7 S. Chakma, L. Das and V. S. Moholkar, Sep. Purif. Technol., 2015, 156, 596-607.

8 J. Guo, Q. Zhang, Z. Cai and K. Zhao, Sep. Purif. Technol., 2016, 161, 69-79.

9 M. T. Yagub, T. K. Sen, S. Afroze and H. M. Ang, Adv. Colloid Interface Sci., 2014, 209, 172-184.

10 M. Abbasi, J. Cleaner Prod., 2017, 145, 105-113.

11 H. Sayğılı and F. Güzel, J. Cleaner Prod., 2016, 113, 995-1004. 
12 V. K. Gupta and Suhas, J. Environ. Manage., 2009, 90, 23132342.

13 G. Crini and P.-M. Badot, Prog. Polym. Sci., 2008, 33, 399-447.

14 L. Cui, Z. Xiong, Y. Guo, Y. Liu, J. Zhao, C. Zhang and P. Zhu, Carbohydr. Polym., 2015, 132, 330-337.

15 M. Vakili, M. Rafatullah, B. Salamatinia, A. Z. Abdullah, M. H. Ibrahim, K. B. Tan, Z. Gholami and P. Amouzgar, Carbohydr. Polym., 2014, 113, 115-130.

16 Y. Ren, H. A. Abbood, F. He, H. Peng and K. Huang, Chem. Eng. J., 2013, 226, 300-311.

17 D. H. K. Reddy and S.-M. Lee, Adv. Colloid Interface Sci., 2013, 201-202, 68-93.

18 L. Zhou, Z. Liu, J. Liu and Q. Huang, Desalination, 2010, 258, 41-47.

19 K. Li, P. Li, J. Cai, S. Xiao, H. Yang and A. Li, Chemosphere, 2016, 154, 310-318.

20 K. Li, Y. Wang, M. Huang, H. Yan, H. Yang, S. Xiao and A. Li, J. Colloid Interface Sci., 2015, 455, 261-270.

21 A. Dolatkhah and L. D. Wilson, ACS Appl. Mater. Interfaces, 2016, 8, 5595-5607.

22 X. Tao, K. Li, H. Yan, H. Yang and A. Li, Environ. Pollut., 2016, 209, 21-29.

23 H. Yan, H. Li, H. Yang, A. Li and R. Cheng, Chem. Eng. J., 2013, 223, 402-411.

24 S. Zhang, Y. Dong, Z. Yang, W. Yang, J. Wu and C. Dong, Chem. Eng. J., 2016, 304, 325-334.

25 X. Li, H. Zheng, Y. Wang, Y. Sun, B. Xu and C. Zhao, Chem. Eng. J., 2017, 319, 119-130.

26 Z. A. Sutirman, M. M. Sanagi, K. J. Abd Karim and W. A. Wan Ibrahim, Carbohydr. Polym., 2016, 151, 1091-1099.

27 L. Song, J. Zhao, H. Yang, J. Jin, X. Li, P. Stagnaro and J. Yin, Appl. Surf. Sci., 2011, 258, 425-430.

28 O. A. Monteiro Jr and C. Airoldi, Int. J. Biol. Macromol., 1999, 26, 119-128.

29 I. A. Udoetok, L. D. Wilson and J. V. Headley, ACS Appl. Mater. Interfaces, 2016, 8, 33197-33209.

30 C. Zhang and A. J. Easteal, J. Appl. Polym. Sci., 2010, 88, 25632569.

31 W. Guo, C. Gao, Q. Li, Z. Cheng and X. Qi, Environ. Prog. Sustainable Energy, 2016, 35, 833-839.
32 R. S. Vieira, M. L. M. Oliveira, E. Guibal, E. RodríguezCastellón and M. M. Beppu, Colloids Surf., A, 2011, 374, 108-114.

33 M. Vandenbossche, M. Jimenez, M. Casetta, S. Bellayer, A. Beaurain, S. Bourbigot and M. Traisnel, React. Funct. Polym., 2013, 73, 53-59.

34 Y. Zhao, J. Li, L. Zhao, S. Zhang, Y. Huang, X. Wu and X. Wang, Chem. Eng. J., 2014, 235, 275-283.

35 X. Liang, J. Duan, Q. Xu, X. Wei, A. Lu and L. Zhang, Chem. Eng. J., 2017, 317, 766-776.

36 L. Huang, S. Yuan, L. Lv, G. Tan, B. Liang and S. O. Pehkonen, J. Colloid Interface Sci., 2013, 405, 171-182.

37 A. Debrassi, A. F. Corrêa, T. Baccarin, N. Nedelko, A. ŚlawskaWaniewska, K. Sobczak, P. Dłużewski, J.-M. Greneche and C. A. Rodrigues, Chem. Eng. J., 2012, 183, 284-293.

38 S. Zhang, Y. Zhang, J. Liu, Q. Xu, H. Xiao, X. Wang, H. Xu and J. Zhou, Chem. Eng. J., 2013, 226, 30-38.

39 X. Sun, L. Yang, Q. Li, J. Zhao, X. Li, X. Wang and H. Liu, Chem. Eng. J., 2014, 241, 175-183.

40 Y. H. Gad, Radiat. Phys. Chem., 2008, 77, 1101-1107.

41 U. Chakraborty, T. Singha, R. R. Chianelli, C. Hansda and P. Kumar Paul, J. Lumin., 2017, 187, 322-332.

42 I. Moreno-Villoslada, C. Torres, F. González, T. Shibue and H. Nishide, Macromol. Chem. Phys., 2009, 210, 1167-1175.

43 Z.-y. Zhang and X.-c. Xu, Chem. Eng. J., 2014, 256, 85-92.

44 R.-L. Tseng, P.-H. Wu, F.-C. Wu and R.-S. Juang, Chem. Eng. J., 2014, 237, 153-161.

45 S. Chatterjee, M. W. Lee and S. H. Woo, Bioresour. Technol., 2010, 101, 1800-1806.

46 S. Saber-Samandari, S. Saber-Samandari, H. Joneidi-Yekta and M. Mohseni, Chem. Eng. J., 2017, 308, 1133-1144.

47 H. Yan, H. Yang, A. Li and R. Cheng, Chem. Eng. J., 2016, 284, 1397-1405.

48 G. Z. Kyzas, D. N. Bikiaris, M. Seredych, T. J. Bandosz and E. A. Deliyanni, Bioresour. Technol., 2014, 152, 399-406.

49 J.-S. Cao, J.-X. Lin, F. Fang, M.-T. Zhang and Z.-R. Hu, Bioresour. Technol., 2014, 163, 199-205.

50 J. Fu, Z. Chen, M. Wang, S. Liu, J. Zhang, J. Zhang, R. Han and Q. Xu, Chem. Eng. J., 2015, 259, 53-61. 